\title{
Benzinli genel amaçlı motorların maksimum güçteki performanslarının karşılaştırılması için basit bir yaklaşım
}

\author{
Emre Arabac1 ${ }^{1 *}$ \\ ${ }^{1}$ Burdur Mehmet Akif Ersoy Üniversitesi, BEGTBMYO, Bucak-Burdur-Türkiye (ORCID: 0000-0002-6219-7246)
}

(İlk Geliş Tarihi 15 Ocak 2019 ve Kabul Tarihi 2 Mart 2019)

(DOI: $10.31590 /$ ejosat.513253)

\begin{abstract}
ATIF/REFERENCE: Arabaci, E. (2019). Benzinli genel amaçlı motorların maksimum güçteki performanslarının karşılaştırılması için
\end{abstract} basit bir yaklaşım. Avrupa Bilim ve Teknoloji Dergisi, (15), 269-279.

\section{$\ddot{O} \mathbf{z}$}

Bahçe çapa makineleri, zincirli testereler, jeneratörler, çim biçme makineleri, mini traktörler gibi makinelerde kullanılan tek veya çift silindirli, düşük güç kapasitesine sahip motorlar genel amaçlı motorlar olarak adlandırılmaktadır. Her motor üreticisinin kendine özgü yapısal tasarımlara sahip motorları bulunmaktadır. Bu motorların güçleri genel olarak kurs hacmine bağlı olarak değişmektedir. Ancak motor katalogları incelendiğinde kurs oranı, maksimum güçteki hızı ve sıkıştırma oranı gibi karakteristik özellikleri birbirinden farklıdır. $\mathrm{Bu}$ çalışmada kurs hacmi, sıkıştırma oranı ve kurs oranı birbirinden farklı olan benzinli genel amaçlı motorların maksimum güçteki performansları, sürtünme, tersinmezlik, egzoz ve 1sı kayıpları da dikkate alınarak karşılaştırılabildiği basit bir termodinamik model sunulmuştur. Sayısal çalışma için 5 farklı üreticiye ait 10 adet genel amaçlı motorun fiziksel özellikleri referans alınarak maksimum güç ürettikleri şartlarda termodinamik modellemeleri yapılarak performansları karşılaştırılmıştır. Motorlara ait kataloglardaki güç değerleri ile termodinamik modele göre hesaplanan güç değerleri birbirine oldukça yakın çıkmıştır ve bu termodinamik modelin kullanılabilir olduğunu göstermektedir. Oluşturulan termodinamik modele göre motorların maksimum çevrim sıcaklıkları, özgül yakıt tüketimleri ve güç yoğulukları karşılaştırılmıştır. Ayrıca her motora ait 1sı balansı oluşturulmuştur. Marka, model ve kurs hacmi ve efektif gücünden bağımsız olarak motor parametreleri kurs oranı ve özgül yakıt tüketimine bağlı olarak incelenmiştir. Yapılan inceleme neticesinde özgül yakıt tüketimi ve kurs oranının artmasıyla birlikte maksimum çevrim sıcaklığı, güç yoğunluğu ve mekanik verimde azalma meydana gelmektedir. Bununla birlikte kurs oranının artması özgül yakıt tüketimini de artırmaktadır. Elde edilen sonuçlara göre motorların karşılaştırılması için yalnızca kurs hacmi ve motor gücü gibi basit özelliklerin yanında kurs oranı, sıkıştırma oranı gibi özelliklerin de karşılaştırma için önemli ve etkili parametreler olduğu görülmüştür. Bu çalışma neticesinde, sunulan termodinamik modelin farklı fiziksel özelliklere sahip benzinli genel amaçlı motorların karşılaştırılabilmesi için kullanılabileceği görülmüştür.

\section{A simple approach for comparing performance of gasoline general- purpose engines at maximum power}

\begin{abstract}
The single or double (twin) cylinder, low power capacity engines used in machines such as garden hoe machines, chainsaws, generators, lawnmowers, mini tractors are called general-purpose engines. Each engine manufacturer has engines with its own unique structural designs. The power of these engines generally varies depending on the swept volume. However, when the engine catalogs are examined, the characteristics such as stroke ratio, maximum power speed, and compression ratio are different. In this study, we present a simple

\footnotetext{
${ }^{*}$ Sorumlu Yazar: Burdur Mehmet Akif Ersoy Üniversitesi, Bucak Emin Gülmez Teknik Bilimler Meslek Yüksekokulu, 15300, Bucak-Burdur-Türkiye, ORCID: 0000-0002-6219-7246 earabaci@,mehmetakif.edu.tr
} 
thermodynamic model in which the gasoline general-purpose engines, whose swept (stroke) volume, compression ratio and stroke ratio are different, can be compared by taking into account their performance at maximum power, friction, irreversibility, exhaust and heat losses. For the numerical study, by comparing the physical properties of 10 general purpose engines of 5 different manufacturers, their performance was compared by performing thermodynamic modeling in the conditions where they produced maximum power. The power values in the catalogs of the engines and the power values calculated according to the thermodynamic model were very close to each other and this thermodynamic model was used. According to the thermodynamic model, maximum cycle temperatures, specific fuel consumption and power density of the engines were compared. In addition, the heat balance of each engines is created. Regardless of the brand, model and stroke volume and effective power, engine parameters are examined according to stroke ratio and specific fuel consumption. As a result of the study, the maximum cycle temperature, power density and mechanical efficiency decrease with the increase in the specific fuel consumption and the stroke ratio. However, the increase in the stroke ratio increases the specific fuel consumption. According to the obtained results, it is seen that the characteristics such as course rate and compression ratio as well as simple properties such as stroke volume and engine power are important and effective parameters for comparison. As a result of this study, it can be seen that the thermodynamic model presented can be used to compare gasoline general purpose engines with different physical properties.

Keywords: General purpose engine, engine performance, Otto cycle, gasoline engines

\section{Giriş}

1678 yılından günümüze kadar içten yanmalı motorlar üzerine birçok çalışma yapılmıştır (Ganesan, 2012). Özellikle 1887 yılındaki Nikolaus August Otto'nun atmosferik gaz motoru patenti (Otto, 1887) ve 1898 yılındaki Rudolf Diesel'in içten yanmalı motor patenti (Diesel, 1898) sonrasında içten yanmalı motorların gelişimi de ivme kazanmıştır. Günümüzde içten yanmalı motorlar hem mobil hem de sabit sistemlerde yaygın bir şekilde kullanılmaktadır (Heywood, 2018).

Düşük güç kapasitelerine sahip kompakt yapılı genel kullanım amaçlı motorlar sıkıştırma ateşlemeli veya buji ateşlemeli olabilmektedir. Bu tür motorlar tek ya da çift silindirli olabilmektedir. Çapa makinesi, zincirli testere ve düşük kapasiteli taşınabilir jeneratörlerde buji ateşlemeli (benzinli) motorlar tercih edilirken, bahçe traktörleri ve yüksek kapasiteli taşınabilir jeneratörlerde ise sıkıştırma ateşlemeli (dizel) motorlar tercih edilmektedir (Arabacı, 2018a).

Isı çevrimleri için yalnızca klasik denge termodinamiği (veya klasik termodinamik) modellerin ötesinde gerçek çevrim kayıplarının da göz önünde bulundurularak daha gerçekçi modellerin oluşturulması istenmektedir. Klasik termodinamikte yer alan 1sı çevrimleri kabulü sanki durağan, başka bir ifade ile 1sıl kayıplar, tersinmezlikler, özgül ısının sıcaklığa bağlı değişimi gibi parametrelerin göz ardı edildiği ve sonsuz sürede gerçekleşen çevrimleridir. Klasik termodinamikte yer alan bu kabullerle gerçek motorların karşılaştırılması çok yanıltıcı olmaktadır. Bu nedenle modern termodinamiğin bir konusu olan sonlu zaman termodinamiği modeli ile teorik çevrimlerin sürtünme, özgül 1sı değişimi, tersinmezlikler, 1sıl kayıplar gibi parametrelerin etkileri dikkate alnınarak sonlu zamanda gerçekleşen çevrim modelleri son zamanlarda sıklıkla kullanılmaktadır (Kaushik ve ark, 2017). Sonlu zaman termodinamiği gaz türbini, absorbsiyon termodinamik çevrimler, iki ısı kaynaklı çevrimler, mikroskobik çevrimler ve içten yanmalı motorlar gibi birçok çevrimi optimize etmek için kullanılabilmektedir (Ge, 2018). Benzinli genel kullanım amaçlı motorların klasik termodinamikteki teorik ısı çevrimi karşılı̆̆ı Otto çevrimidir (Wu, 2002). Bu çevrimde süreçler tersinirdir ve çalışma maddesi olarak ideal hava ve sabit özgül ısılar kullanılmaktadır. Bununla birlikte, sürtünme, 1sı transferi gibi kayıplar ihmal edilmektedir. Bu nedenle teorik 1sı çevrimlerinin 1sıl verimleri gerçek motorların 1sıl verimlerinden oldukça yüksek olmaktadır (Ansermet, 2019).

Son yıllarda sonlu zaman termodinamiği yaklaşımı kullanılarak içten yanmalı motorların modellendiği birçok çalışma yapılmıştır. Bu tür çalışmalarda sıkıştırma oranı (Gonca ve ark., 2016, Ebrahimi ve ark., 2018, Ebrahimi ve ark., 2013), kurs uzunluğu (Ebrahimi, 2012, Gonca ve ark., 2016, Dobrucal1, 2016), kurs oranı (Gonca ve ark., 2013), sürtünme kayb1 (Ebrahimi, 2011, Gonca ve ark., 2016), yanma verimi (Wu ve ark. 2018), 1sil verim (Ge ve ark., 2018, You ve ark. 2018), termodinamik verim (Arabacı, 2018b), artık gazların oranı (Ebrahimi, 2013, Özdemir, 2018), 1sı transferi kayıpları (Ge ve ark., 2017), entropi üretimi (You ve ark., 2017, Ebrahimi, 2016) gibi birçok parametrenin etkileri tartışılmıştır. Ayrıca yapılan çalışmalarda çalışma maddesi olarak sadece hava (Ge ve ark., 2017, Ge ve ark., 2005), hava-yakıt karışımı (Gonca, 2017, Ebrahimi, 2016) veya hava-yakıt-artık gaz karışımı (Ebrahimi, 2013) da kullanılmıştır. Çevrimdeki çalışma maddesinin özgül ısısı için sabit (Ebrahimi, 2013, Özdemir ve ark., 2018), sıcaklığa bağlı lineer değişen (Wu ve ark., 2018, Ge ve ark., 2017), veya sıcaklığa bağlı polinomal değişen (Ge ve ark., 2018) özgül 1sı kabulü ile modeller oluşturulmuştur. Ayrıca sonlu zaman termodinamiği, Otto (Ge ve ark., 2018, Ge ve ark., 2017), Diesel (Ge ve ark., 2008), Karma (Ge ve ark. 2009), Miller (Wu ve ark., 2018, Wu ve ark., 2017), Atkinson (Ebrahimi, 2011) gibi birçok içten yanmalı motor çevriminin optimizasyonu, karşılaştırılması ve tasarım parametrelerinin incelenmesi için uygulanmaktadır.

Dobrucalı ve arkadaşları, tersinmez bir Otto-Miller çevrimi için 1sı transferi, sürtünme, sıkıştırma ve genişleme işlemlerindeki iç tersinmezlikler ve özgül ısının sıcaklığa bağlı değişimi göz önünde bulundurularak termodinamik bir analiz yapmışlardır. Yapılan analizde çevrim sıcaklık oranı, çevrim basınç oranı, sürtünme katsayısı, motor hızı, ortalama piston hızı, kurs uzunluğu, giriş sıcaklı̆̆1 giriş basınc1, eşdeğerlik oranı, sıkıştırma oranı ve kurs oranı gibi motor tasarım parametrelerinin efektif güce, efektif güç yoğunluğuna ve efektif verime olan etkileri detaylı bir şekilde incelenmiştir (Dobrucalı ve ark., 2016). Ge ve arkadaşları, tersinmez Otto çevrimi için hava standardı varsayımı ile sürtünme, iç tersinmezlikler ve sürtünme kaybının etkileri göz önünde bulundurularak termodinamik bir model olulturmuşlardır. Oluşturulan modelde entropi üretim oranı ve ekolojik fonksiyon parametreleri, motor performans parametrelerine göre detaylı olarak incelenmiştir (Ge ve ark., 2018). Ebrahimi ve arkadaşları, tersinmez hava standardı dual çevriminde özgül ısı değişiminin, sürtünme kayıplarının, iç tersinmezliklerinin ve ısı transferi kayıplarının etkilerinin kurs uzunluğu ve hacimsel 
verimin etkilerinin incelendiği termdinamik bir model oluşturmuştur. Bu model ile birlikte kurs uzunluğu ve hacimsel verimin motor performans parametrelerine olan etkileri sayısal örnekle birlikte detaylı bir şekilde incelenmiştir (Ebrahimi ve ark., 2013). Özdemir ve arkadaşları, ortalama piston hızı ve artık gaz oranının Otto çevrimli motor performansı üzerindeki etkisinin incelenmesi için tersinmezliklerin hesaba katıldığı termodinamik bir model oluşturulmuştur. Oluşturulan modelde çevrim çalışma maddesi olarak yakıthava-artık gaz karışımı kullanılmıştır. Ayrıca çevrim başlangıç sıcaklığı, artık gaz miktarı ve sıcaklığının bir fonksiyonu olarak ifade edilmiştir (Özdemir ve ark., 2018). Ebrahimi, hava standardı Atkinson çevrimi performansını özgül ısı oranının sıcaklığa bağlı doğrusal olarak değiştiğini, sürtünme kaybının piston ortalama hızına göre değiştiği ve iç tersinmezliklerin hesaba katıldığı bir termodinamik model oluşturmuştur. Oluşturulan termodinamik model güç çıkışı- sıkıştırma oranı, güç çıkışı-1sıl verim arasındaki ilişkiler detaylı sayısal örnekle ifade edilmiştir (Ebrahimi, 2011). Ge ve arkadaşları, hava standardı miller çevrimi için sısı transferi ve sürtünme gibi kayıpların hesaba katıldığı termodinamik bir model oluşturmuşlardır. Yapılan çalışmada, güç çıkışı-sıkıştırma oranı ve 1sıl verimsıkıştırma oranı arasındaki ilişkiler sayısal örnekle sunulmuş ve tartışılmıştır. (Ge ve ark., 2005). You ve arkadaşları, politropik süreç ve 1S1 transferi kayıplarının hesaba katıldığı dual-Miller çevrimi ile ilgili termodinamik bir model oluşturmuşlardır. Entropi üretim oranı, ekolojik faktör gibi ikinci yasa parametreleri sonlu zaman termodinamiği ile ele alınmıştır. Sayısal hesaplamalar neticesinde sıkıştırma oranı, kesme oranı ve politropik üslerin değişiminin motor performansı üzerindeki etkileri termodinamik olarak analiz edilmiştir (You ve ark., 2015). Ge ve arkadaşları, Ge ve arkadaşları, iç tersinmezlikler, sürtünme kaybı ve ısı transferi kaybının hesaba katıldığı hava standardı otto çevrimi için termodinamik modeli oluşturmuşlardır. Oluşturulan model ile birlikte entropi üretim oranı ve ekolojik faktör parametreleri tersinmezlik kaynaklarına göre motor performans parametrelerine olan etkileri incelenmiştir. (Ge ve ark., 2017).

Bu çalışmada farklı yapısal özelliğe ve farklı kapasiteye sahip hava soğutmalı, normal emişli ve benzinli genel amaçlı motorların tam yükteki (maksimum güçteki) performanslarının karşılaştırılabildiği bir model oluşturulmuştur. Bunun için öncelikle farklı üreticilere ait motorların teknik katalogları incelenerek, sıkıştırma oranı, maksimum güç, maksimum güçteki hız, kurs/çap oranı ve kurs hacmi değerleri elde edilmiştir. Hesaplamalarda bu değerler referans olarak kabul edilmiştir. Sonlu zaman termodinamiği modeli için çalışma maddesinin stokiyometrik oranda hava-yakıt ve belirli oranda önceki çevrimden silindir içerisinde kalan artık gaz olduğu kabul edilmiştir. Özgül ısıların sıcaklığa bağlı olarak logaritmik bir fonksiyonda değiştiği kabul edilmiştir.

\section{Termodinamik Model}

Şekil 1'de gösterilen Otto çevrimine ait $P V$ ve $T S$ diyagramında 0-1 ve 1-0 ise sırasıyla emme ve egzoz zamanını, "s" indisi ise tersinir olarak gerçekleşen sıkıştırma ve genleşme süreçlerini ifade etmektedir. (Arabacı, 2018b).
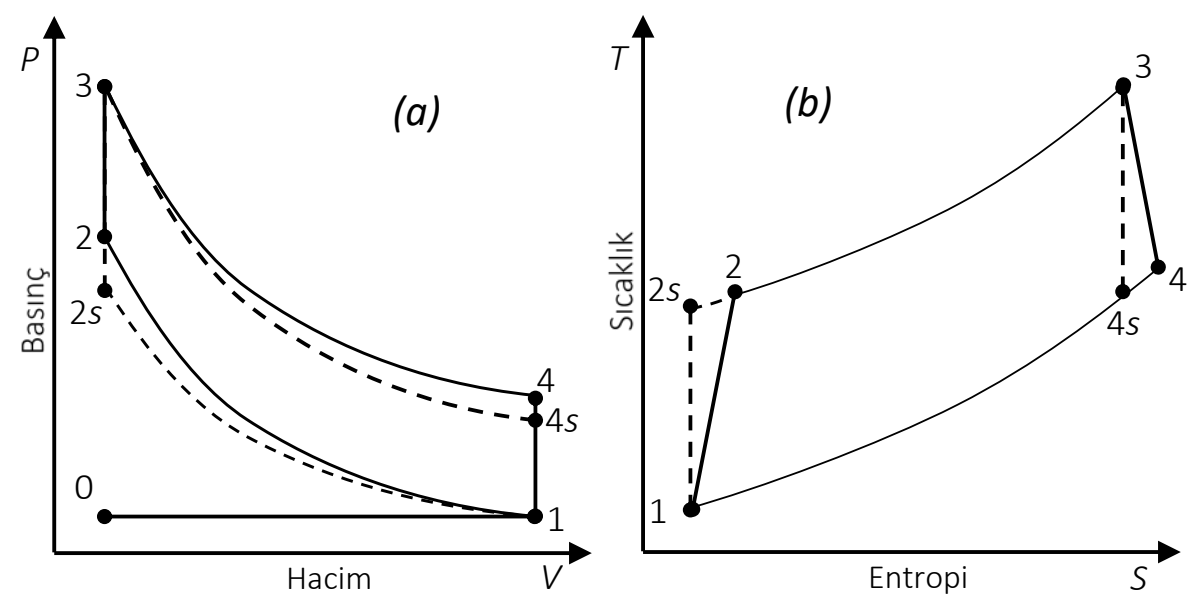

Şekil 1. Otto çevrimi için (a) PV ve (b) TS diyagramı

Otto çevriminde $2 \rightarrow 3$ sürecinde gerçekleşen birim zamandaki ısı girişi $\left(\dot{Q}_{i n}\right)$ ile $4 \rightarrow 1$ sürecinde gerçekleşen birim zamandaki çıkan 1Sı $\left(\dot{Q}_{\text {out }}\right)$ arasındaki fark indike gücü $\left(P_{i}\right)$ ifade etmektedir. Efektif güç $\left(P_{e}\right)$ ise $P_{i}$ ile sürtünme gücünün $\left(P_{f r}\right)$ arasındaki farka eşittir. $P_{e} / P_{i}$ oranı ise mekanik verim $\left(\eta_{m}\right)$ olarak tanımlanmaktadır.

$$
\begin{gathered}
P_{i}=Q_{\text {in }}-\left|Q_{\text {out }}\right| \\
P_{e}=P_{i}-P_{f r} \\
\eta_{m}=\frac{P_{e}}{P_{i}}=\frac{Q_{\text {in }}-\left|Q_{\text {out }}\right|-\left|P_{f r}\right|}{Q_{\text {in }}-\left|Q_{\text {out }}\right|}=1-\frac{\left|P_{\text {fr }}\right|}{Q_{\text {in }}-\left|Q_{\text {out }}\right|}=\frac{P_{e}}{P_{e}+\left|P_{f r}\right|}
\end{gathered}
$$

$P_{f r}$, sürtünme katsayısı $(\mu)$ ve ortalama piston hızının $\left(s_{p m}\right)$ bir fonksiyonu olarak aşağıdaki gibi ifade edilmektedir.

$$
P_{f r}=-\mu\left(s_{p m}\right)^{2}=\frac{-\mu N^{2}}{900}\left[\frac{4 \lambda^{2} v_{d}}{\pi}\right]^{2 / 3}
$$


Burada $N, \lambda$ ve $v_{d}$ sırasıyla motor hızı, kurs oranı ve kurs hacmini ifade etmektedir. Sistemdeki çalışma akışkanının $\left(m_{m i x}\right)$ yakıt $\left(m_{f}\right)$, hava $\left(m_{a}\right)$ ve artık gazdan $\left(m_{r}\right)$ meydana geldiği varsayılmıştır. Artık gazın ise $C_{8} H_{18}$ 'in stokiyometrik orandaki hava ile tam yanma sonucundaki $\mathrm{CO}_{2}, \mathrm{H}_{2} \mathrm{O}$ ve $\mathrm{N}_{2}$ den oluşan bir karışımından meydana geldiği kabul edilmiştir (Özdemir ve ark., 2018).

$$
\begin{gathered}
m_{\text {mix }}=m_{f}+m_{a}+m_{r}=\frac{m_{f}(\phi+\alpha)}{\phi\left(1-x_{r}\right)} \\
\dot{m}_{\text {mix }}=\frac{m_{\text {mix }} N}{120}
\end{gathered}
$$

Burada $\phi, \alpha$ ve $x_{r}$, sırasıyla eşdeğerlik oranını, stokiyometrik hava/yakıt oranını ve artık gaz kesrini ifade etmektedir. $\lambda$ ve $x_{r}$ aşă̆ıdaki gibi tanımlanmıştır.

$$
\begin{gathered}
\lambda=\frac{L}{D}=\frac{4 v_{d}}{\pi D^{3}} \\
x_{r}=\frac{m_{r}}{m_{\text {mix }}}
\end{gathered}
$$

Çevrim başlangıç sıcaklığı olan $T_{1}$ genellikle ortam sıcaklığına eşit ve sabit bir değer olarak kabul edilmektedir. Bu çalışmada ise $T_{1}$ değeri $T_{a}, T_{4}$ ve $x_{r}$ 'nin parametresi olarak aşağıdaki gibi ifade edilmiştir:

$$
T_{1}=T_{a}+\frac{x_{r} R_{r}\left(T_{4}-T_{a}\right)}{R_{m i x}}
$$

$\dot{Q}_{\text {in }}$ ve $\dot{Q}_{\text {out }}$ ise aşağıdaki gibi ifade edilmiştir.

$$
\begin{gathered}
\dot{Q}_{\text {in }}=\dot{m}_{\text {mix }} \int_{T_{2}}^{T_{3}} c_{v m i x} d T \\
\dot{Q}_{\text {out }}=\dot{m}_{\text {mix }} \int_{T_{4}}^{T_{1}} c_{v \text { mix }} d T
\end{gathered}
$$

Bu çalışmada özgül ısıların sıcaklığın logaritmik bir fonksiyonu olduğu kabul edilmiştir. Bunun için NIST-JANAF termokimyasal katsayılarından (Chase, 1998) faydalanılarak yakıt, hava ve artık gaz için özgül ısının sıcaklığa bağlı değişimi eğrisi elde edilmiş ve bu eğri $y=a+b \ln x$ genel formundaki logaritmik bir fonksiyonla ifade edilmiştir.

$$
\begin{gathered}
c_{v i}=a_{v i}+b_{i} \ln T \\
c_{p i}=a_{p i}+b_{i} \ln T \\
R_{i}=a_{p i}-a_{v i}
\end{gathered}
$$

Buradaki “i” indisi hava $(a)$, yakıt $(f)$, artık gaz $(r)$ veya çalışma akışkanını (mix) ifade etmektedir. Buradaki katsayılar tablo 1'deki gibi belirlenmiştir.

Tablo 1. NIST-JANAF tablolarl yardimiyla türetilen katsayllar

\begin{tabular}{c|ccc}
\hline Katsay 1 & Yakıt & Hava & Artık Gaz \\
\hline$a_{v i}$ & -8.31762 & -0.12911 & -0.41814 \\
$a_{p i}$ & -8.24469 & 0.15917 & -0.12737 \\
$b_{i}$ & 1.75018 & 0.14370 & 0.20460 \\
\hline
\end{tabular}

Herhangi bir sıcaklık için çalışma akışkanına ait $a_{v m i x}, a_{p m i x}$ ve $b_{m i x}$ katsayıları aşağıdaki gibi hesaplanabilmektedir.

$$
\begin{aligned}
a_{v m i x} & =\frac{1-x_{r}}{1+\alpha \lambda}\left(a_{v f}+\alpha x_{r} a_{v a}\right)+x_{r} a_{v r} \\
a_{p m i x} & =\frac{1-x_{r}}{1+\alpha \lambda}\left(a_{p f}+\alpha x_{r} a_{p a}\right)+x_{r} a_{p r} \\
b_{\text {mix }} & =\frac{1-x_{r}}{1+\alpha \lambda}\left(b_{f}+\alpha x_{r} b_{a}\right)+x_{r} b_{r}
\end{aligned}
$$

Buna göre Eş. 10 ve Eş. 11 aşağıdaki gibi tekrar ifade edilebilmektedir.

$$
\dot{Q}_{\text {in }}=\dot{m}_{\text {mix }} b_{\text {mix }}\left[\left(T_{3} \ln T_{3}-T_{2} \ln T_{2}\right)-\left(T_{3}-T_{2}\right)\left(1-\frac{a_{v m i x}}{b_{\text {mix }}}\right)\right]
$$




$$
\left|\dot{Q}_{\text {out }}\right|=\dot{m}_{\text {mix }} b_{\text {mix }}\left[\left(T_{4} \ln T_{4}-T_{1} \ln T_{1}\right)-\left(T_{4}-T_{1}\right)\left(1-\frac{a_{\text {vmix }}}{b_{\text {mix }}}\right)\right]
$$

$1 \rightarrow 2 s$ ve $3 \rightarrow 4 s$ süreçleri tersinir adyabatik sıkıştırma ve genişleme süreçlerini ifade etmektedir. 2 ve 4 noktalarının termodinamik özelliklerinin belirlenebilmesi için öncelikle $2 s$ ve $4 s$ noktalarının termodinamik özellikleri belirlenmelidir.

$$
\begin{gathered}
c_{v \text { mix }} \frac{d T}{T}+R_{\text {mix }} \frac{d v}{v}=0 \\
\int_{T_{1}}^{T_{2 s}}\left(\frac{a_{v \text { mix }}+b_{\text {mix }} \ln (T)}{T}\right) d T=-R_{\text {mix }} \int_{V_{1}}^{V_{2 s}} \frac{d V}{V} \\
\int_{T_{3}}^{T_{4 s}}\left(\frac{a_{v \text { mix }}+b_{\text {mix }} \ln (T)}{T}\right) d T=-R_{\text {mix }} \int_{V_{3}}^{V_{4 s}} \frac{d V}{V}
\end{gathered}
$$

Burada tersinir adyabatik süreçler için genel ifade Eş. 20 ile ifade edilmiştir. $1 \rightarrow 2 s$ ve $3 \rightarrow 4 s$ süreçleri için Eş. 20 düzenlenirse Eş. 21 ve Eş 22 elde edilir. Bu eşitlikler çözüldüğünde $T_{2 s}$ ve $T_{4 s}$ aşağıdaki gibi ifade edilmektedir.

$$
\begin{aligned}
& \left.T_{2 s}=e^{\left[\frac{-1}{b_{\text {mix }}}\left[a_{v_{\text {mix }}}-\left(\left(a_{v \text { mix }}\right)^{2}+b_{\text {mix }}\left(b_{\text {mix }}\left(\ln T_{1}\right)^{2}+2 a_{v \text { mix }} \ln T_{1}+2 R_{\text {mix }} \ln \varepsilon\right)\right)^{0.5}\right]\right.}\right] \\
& \left.T_{4 s}=e^{\left[\frac{-1}{b_{\text {mix }}}\left[a_{\text {vmix }}-\left(\left(a_{v \text { mix }}\right)^{2}+b_{\text {mix }}\left(b_{\text {mix }}\left(\ln T_{3}\right)^{2}+2 a_{\text {vmix }} \ln T_{3}-2 R_{\text {mix }} \ln \varepsilon\right)\right)^{0.5}\right]\right.}\right]
\end{aligned}
$$

Sıkıştırma ve genişleme süreçleri için tersinmezlikler sırasıyla sıkıştırma verimi $\left(\eta_{c}\right)$ ve genleşme verimi $\left(\eta_{e}\right)$ ile ifade edilmektedir ve izentropik verim olarak bilinmektedir. (Özdemir ve ark., 2018). $\eta_{c}$ ve $\eta_{e}$ bu çalışma için \%97 olarak kabul edilmiştir (Arabacı, 2018a). İzentropik verimler yardımıyla $T_{2}$ ve $T_{4}$ aşağıdaki gibi ifade edilmektedir.

$$
\begin{gathered}
T_{2}=\frac{T_{2 s}-T_{1}}{\eta_{c}}+T_{1} \\
T_{4}=\eta_{e}\left(T_{4 s}-T_{3}\right)+T_{3}
\end{gathered}
$$

Bu çalışmada çalışma akışkanı olarak hava yerine hava-yakıt-artık gaz karışımı kullanıldığından dolayı $\dot{Q}_{\text {in }}$ aynı zamanda yakıt kimyasal enerjisi $\left(\dot{Q}_{f}\right)$ ve silindir duvarından asılan 1sı $\left(\dot{Q}_{h t}\right)$ ile ifade edilmektedir.

$$
\dot{Q}_{i n}=\dot{Q}_{f}-\dot{Q}_{h t}
$$

$\dot{Q}_{h t}$ çevrimdeki tersinmezlik kaynaklarından birisidir ve aşağıdaki gibi ifade edilmektedir.

$$
\begin{gathered}
\dot{Q}_{h t}=\alpha_{c o n v} A_{t r}\left(\frac{T_{2}+T_{3}}{2}-T_{w}\right) \\
A_{t r}=\pi D\left[\frac{\varepsilon L}{\varepsilon-1}+\frac{D}{2}\right]=\pi\left(\frac{4 v_{d}}{\pi \lambda}\right)^{2 / 3}\left[\frac{\varepsilon \lambda}{(\varepsilon-1)}+0.5\right] \\
\alpha_{c o n v}=130\left(\frac{\varepsilon v_{d}}{\varepsilon-1}\right)^{-0.06} P_{1}^{0.8} T_{1}^{-0.4}\left(s_{p m}+1.4\right)^{0.8} \\
s_{p m}=\frac{L N}{30}=\frac{D \lambda N}{30}=\frac{2}{15}\left[\frac{N^{3} \lambda^{2} v_{d}}{\pi}\right]^{1 / 3}
\end{gathered}
$$

Burada $\alpha_{c o n v}, A_{t r}, T_{w}$ sırasıyla ısı transferi katsayısı, ısı transferi yüzey alanı ve silindir duvar sıcaklığıdır. $\alpha_{\text {conv }}$ genellikle sabit bir değer olarak kabul edilmektedir. Ancak bu çalışmada Hohenberg korelasyonu kullanılmıştır (Hohenberg, 1979).

$\dot{Q}_{f}$ ise $\phi$ ve yakıt alt ısıl değerinin $\left(H_{u}\right)$ bir fonksiyonu olarak aşağıdaki amprik ifade ile hesaplanabilmektedir (Ebrahimi, 2012).

$$
\dot{Q}_{f}=\left(-1.4474+\frac{4.1858}{\phi}-\frac{1.8671}{\phi^{2}}\right) \dot{m}_{f} H_{u}
$$

Yukarıdaki eşitlikler $P_{e}$ değerinin hesaplanması için yeterlidir ancak yalnızca $P_{e}$ değerini kullanarak farklı güç kapasitesindeki motorların karşılaştırılması için yeterli değildir. Bu çalışmada motorların karşılaştırılması için ısıl verim, güç yoğunluğu (birim kurs hacmi başına elde edilen güç, $\left.P_{d e n}\right)$ ve özgül yakıt tüketimi $\left(b_{e}\right)$ parametreleri kullanılmıştır.

$$
P_{d e n}=\frac{10^{3} P_{e}(\varepsilon-1)}{\varepsilon v_{d}}
$$




$$
b_{e}=\frac{3.6 \times 10^{6} \dot{m}_{f}}{P_{e}}
$$

Genel amaçlı motorlarda marka, model ve kapasiteye bağlı olarak $\varepsilon, \lambda, v_{d}$ ve $N$ gibi fiziksel özellikleri birbirinden farklı olabilmektedir. $P_{1}, T_{0}, T_{w}, \mu, H_{u}, \phi$ gibi diğer parametreler ise her motor için sabit olarak kabul edilmiştir.

\section{Sayısal Uygulama ve Tartışma}

Bu çalışma için 5 farklı üreticiye ait 10 adet genel amaçlı motorun karakteristik özellikleri referans alınarak maksimum güç ürettikleri şartlarda matematiksel modellemeleri yapılarak performansları karşılaştırılmışıı (Tablo 2). Bu motorların maksimum güçleri 4.2-10.3 kW, sıkıştırma oranları, 8.1-8.7, kurs oranları 0.716-0.970 ve kurs hacimleri 169-479 $\mathrm{cm}^{3}$ aralığında değişmektedir. Tablo 2'de yâre alan $P_{e, \max }$ değerleri, motorların süreklü maksimum güç değerleridir. Ayrıca $\lambda$ deperleri kataloglardaki kurs boyu ve piston çapı verilerinden hesaplanmıştır.

Tablo 2. Saylsal uygulamada kullanilan motorlar

\begin{tabular}{c|cccc}
\hline Marka-Model & $\begin{array}{c}v_{d} \\
\mathrm{~cm}^{3}\end{array}$ & $\begin{array}{c}P_{e, \max } \\
\text { kW@rmp }\end{array}$ & $\varepsilon$ & $\lambda$ \\
\hline Honda GX270 & 270 & $6.3 @ 3600$ & 8.2 & 0.753 \\
Honda GX390 & 389 & $8.7 @ 3600$ & 8.2 & 0.727 \\
Lombardini LGA 280 & 275 & $6.3 @ 3800$ & 8.5 & 0.865 \\
Lombardini LGA 340 & 338 & $8.1 @ 3800$ & 8.5 & 0.780 \\
Subaru EX40 & 404 & $10.3 @ 3600$ & 8.3 & 0.730 \\
Subaru EX17 & 169 & $4.2 @ 4000$ & 8.5 & 0.716 \\
Vanguard 8 & 305 & $6.0 @ 3600$ & 8.1 & 0.782 \\
Vanguard 13 & 479 & $9.7 @ 3600$ & 8.2 & 0.970 \\
Yamaha MZ360 & 357 & $7.6 @ 3600$ & 8.1 & 0.741 \\
Yamaha MZ250 & 253 & $5.4 @ 3600$ & 8.7 & 0.797 \\
\hline
\end{tabular}

Hesaplamalarda kullanılan sabit parametrelerin değerleri Tablo 3’te gösterilmiştir (Arabacı, 2018a).

Tablo 3. Hesaplamalarda kullanilan sabit parametreler

\begin{tabular}{c|c}
\hline Parametre & Değer \\
\hline$P_{1}$ & $100 \mathrm{kPa}$ \\
$T_{a}$ & $300 \mathrm{~K}$ \\
$T_{w}$ & $350 \mathrm{~K}$ \\
$\mu$ & $12.9 \mathrm{Ns} / \mathrm{m}$ \\
$H_{u}$ & $44700 \mathrm{~kJ} / \mathrm{kg}$ \\
$\phi$ & 1.0 \\
$x_{r}$ & $\% 13$ \\
\hline
\end{tabular}

Şekil 2'de referans motorlara ait performans verileri gösterilmektedir. Şekil 2a'da matematiksel modele göre hesaplanan ve motor kataloglarında bulunan maksimum güç değerlerinin karşılaştırması yapılmıştır. Matematiksel model ile elde edilen maksimum güç sonuçlarının katalog değerlerine çok yakın olduğu görülmektedir. Şekil 2b'de çevrimde oluşan maksimum sıcaklıklar $\left(T_{3}\right)$ görülmektedir. Teorik olarak $T_{3}$ sıkıştırma sonu sıcaklığı olan $T_{2}$ ve $\dot{Q}_{i n}$ 'e bağlıdır. $T_{2}$ ise $T_{1}, \varepsilon$ ve $\eta_{c}$ 'ye bağlıdır. $Q_{i n}$ ise $b_{e}$ 'ye bağlı olarak değişmektedir. Bu nedenle $T_{3}$ ve $b_{e}$ 'nin aynı eğilimde olması beklenmektedir. Ancak Şekil $2 \mathrm{~b}$ ve Şekil $2 \mathrm{c}$ beraber incelendiğinde $T_{3}$ 'ün en yüksek olduğu durumda $b_{e}$ 'nin en düşük olduğu görülmektedir. Sunulan modelde $\dot{Q}_{i n}, \dot{Q}_{f}$ ve $\dot{Q}_{h t}$ 'ye bağlıdır. $\dot{Q}_{h t}$ ise $T_{1}, T_{2}$, $T_{3}, \varepsilon, v_{d}$, ve $\lambda$ gibi birçok parametreye bağlıdır. $b_{e}$ ise yalnızca $\dot{Q}_{f}$ ile ilgili bir durumdur ve aynı $\dot{Q}_{f}$ değerinde $\dot{Q}_{h t}$ 'nin değişimine bağlı olarak $\dot{Q}_{\text {in }}$ değişebilir. Bu durumda motorda, başta $T_{3}$ olmak üzere tüm kritik değerler değişmektedir. Bu matematiksel modelde $T_{3}$ herhangi bir formüle göre doğrudan olarak hesaplanamaz ve iteratif yöntemlerle belirlenmesi gerekmektedir. Şekil $2 \mathrm{~d}^{\prime}$ 'de ise güç yoğunluğu görülmektedir. Güç yoğunluğu hacimsel bir kavramdır ve genel olarak $b_{e}$ 'nin veya $T_{3}$ 'ün veya $P_{e}$ 'nin değişimiyle birikte güç yoğunluğunun nasıl değişeceği konusuda doğrudan bir ilişki kurmak oldukça zordur. Şekil 2e'de her motor için 1sı balansı oluşturulmuştur. Isı balansındaki değerler yüzde olarak verildiği için karşılaştırma yapılabilir niteliktedir. 


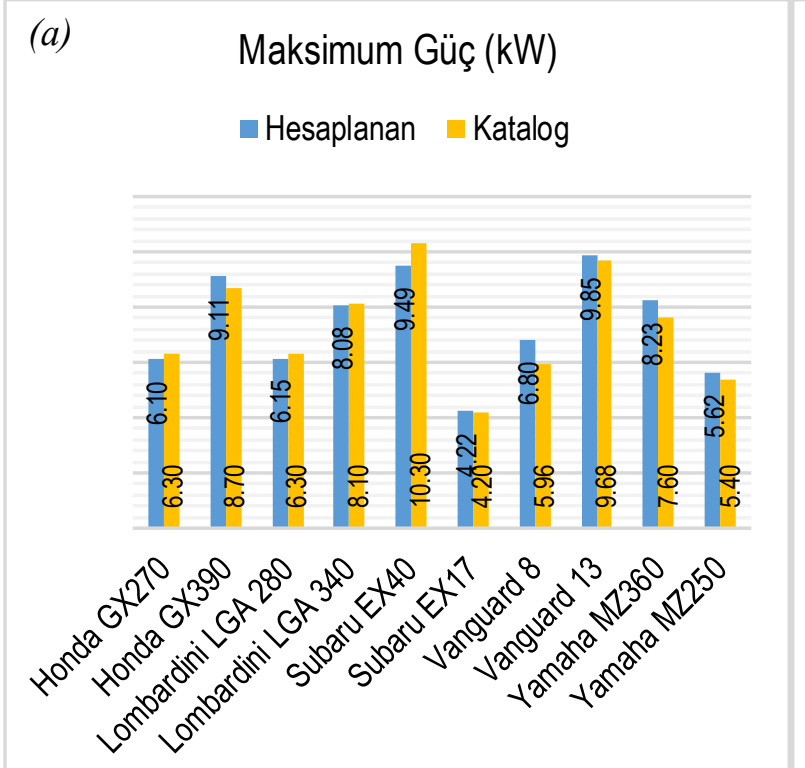

(b) Maksimum Sicaklk (K)

(c) Özgül Yakıt Tüketimi (g/kWh)

(d)
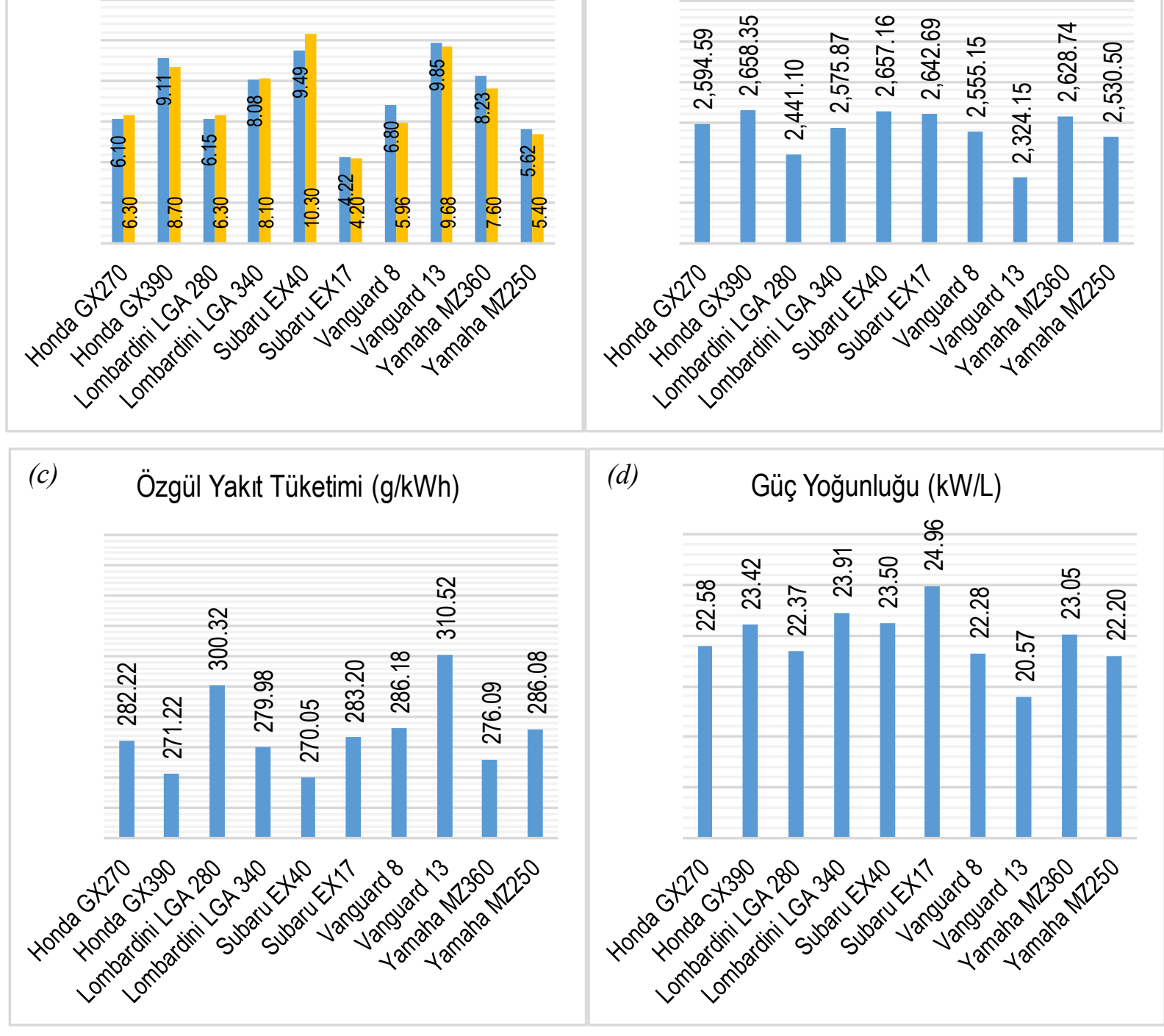

(e)

Isı Balansı (\%)

घfektif Güç $\quad$ Egzoz Kayıp Gücü $\quad$ घ Isı Transferi Kayıp Gücü $\quad$ - Sürtünme Kayıp Gücü

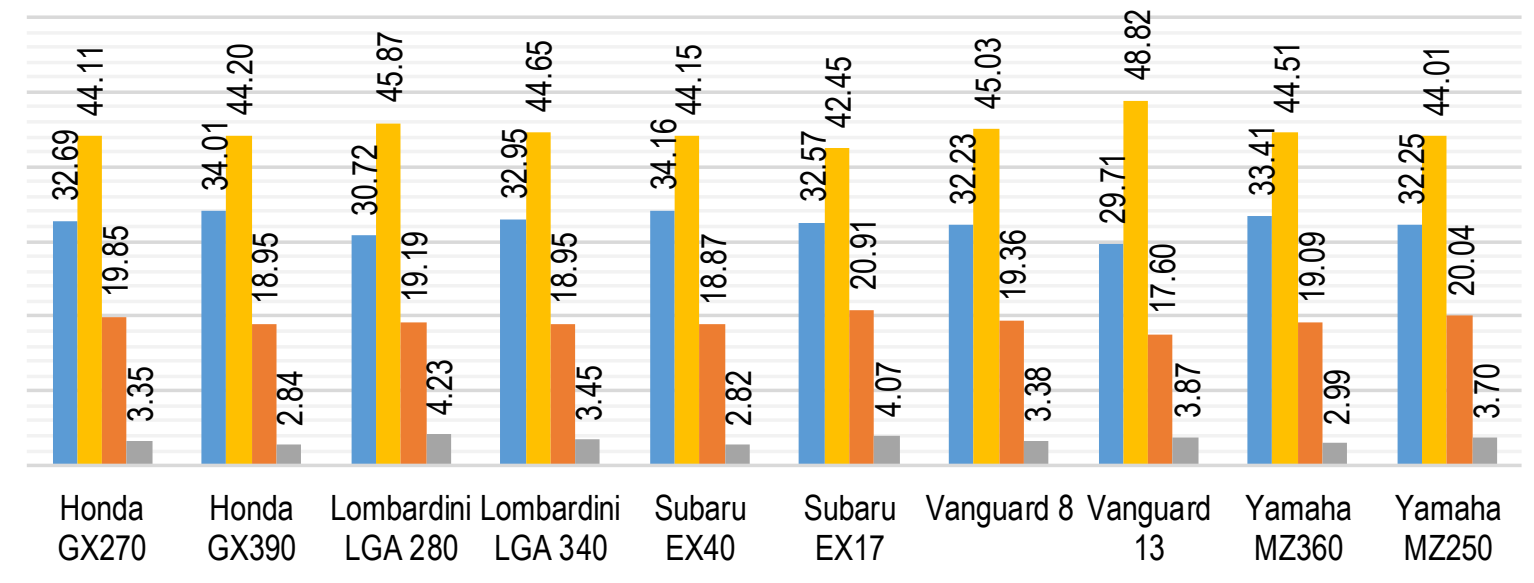

Şekil 2. Referans motorlara ait performans verilerinin karşılaş̧tırlması

(a) Maksimum Gü̧,, (b) Maksimum Sicaklık, (c) Özgül Yakıt Tüketimi, (d) Gü̧̧ Yoğunluğu, (e) Isı Balansı 
Marka, model ve kurs hacmi ve efektif gücünden bağımsız olarak motor parametreleri $\lambda$ ve $b_{e}$ 'ye bağl olarak incelenmiştir. Şekil 3 ’te kurs oranının $(\lambda)$ özgül yakıt tüketimine $\left(b_{e}\right)$ ve güç yoğunluğuna $\left(P_{\text {dens }}\right)$ etkisi görülmektedir. Kurs oranının artmasıyla birlikte özgül yakıt tüketiminin arttığı ve güç yoğunluğunun azaldığı görülmektedir. Başka bir ifade ile kurs oranı düşük olan motorun özgül yakıt tüketimi düşük, güç yoğunluğu yüksek olmaktadır.
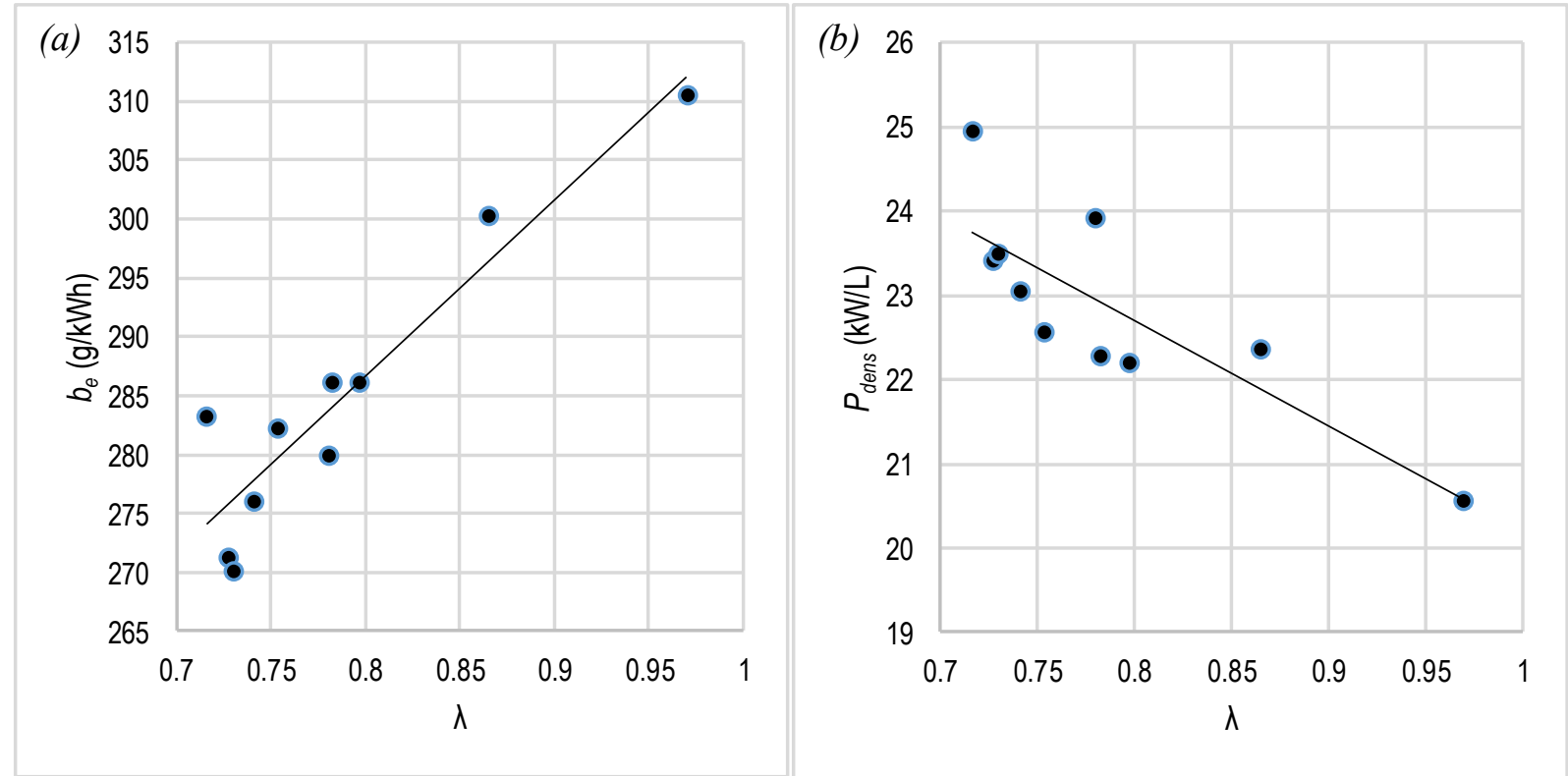

Şekil 3. (a) Özgül yakıt tüketiminin ve (b) güç yoğunluğunun $\lambda$ ‘ya bağlı değişimi

Şekil 4 'te kurs oranının $(\lambda)$ maksimum sıcaklığa $\left(T_{3}\right)$ ve mekanik verime $\left(\eta_{m}\right)$ etkisi görülmektedir. Kurs oranı arttıkça maksimum sicaklık ve mekanik verim azalmaktadır. Kurs oranı $\dot{Q}_{h t}, s_{p m}$ ve $P_{f r}$ 'yi etkilemektedir. Bu nedenle kurs oranı $(\lambda)$ arttıkça $\dot{Q}_{h t}$ 'ye bağlı olarak $T_{3}, P_{f r}$ 'ye bağlı olarak $\eta_{m}$ olumsuz etkilenmektedir. Ancak $\lambda$ ile $T_{3}$ arasındaki ilişki $\lambda$ ile $\eta_{m}$ arasındaki ilişkiden çok daha güçlü olduğu görülmektedir.

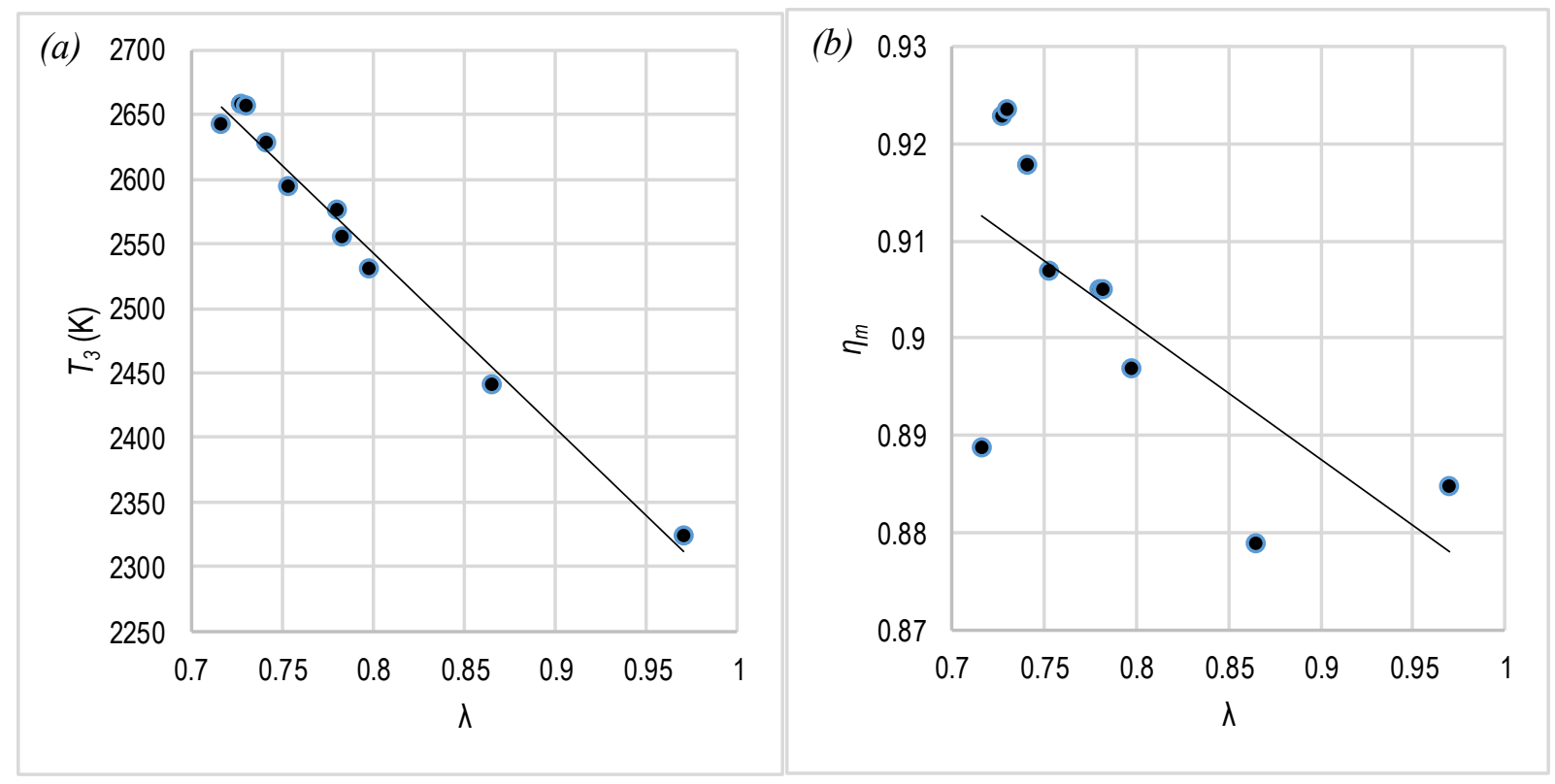

Şekil 4. (a) Maksimum sıcaklı̆̆ın ve (b) mekanik verimin $\lambda$ 'ya bağlı değişimi

Şekil 5'te özgül yakıt tüketiminin $\left(b_{e}\right)$ güç yoğunluğuna $\left(P_{\text {dens }}\right)$ ve maksimum sıcaklığa $\left(T_{3}\right)$ etkisi görülmektedir. $b_{e}$ arttıkça $P_{d e n s}$ ve $T_{3}$ azalmaktadır. İçten yanmalı motolarda $b_{e}$ 'nin mümkün olduğunca düşük olması istenmektedir. $b_{e}$ azaldığında $P_{\text {dens }}$ beklenen bir durum olarak artmaktadır ve bu da istenen bir durumdur. Ancak $b_{e}$ azaldığında $T_{3}$ artmaktadır ve bu durumda motorun daha yüksek sıcaklıklarda çalıştı̆ıııı göstermektedir. 

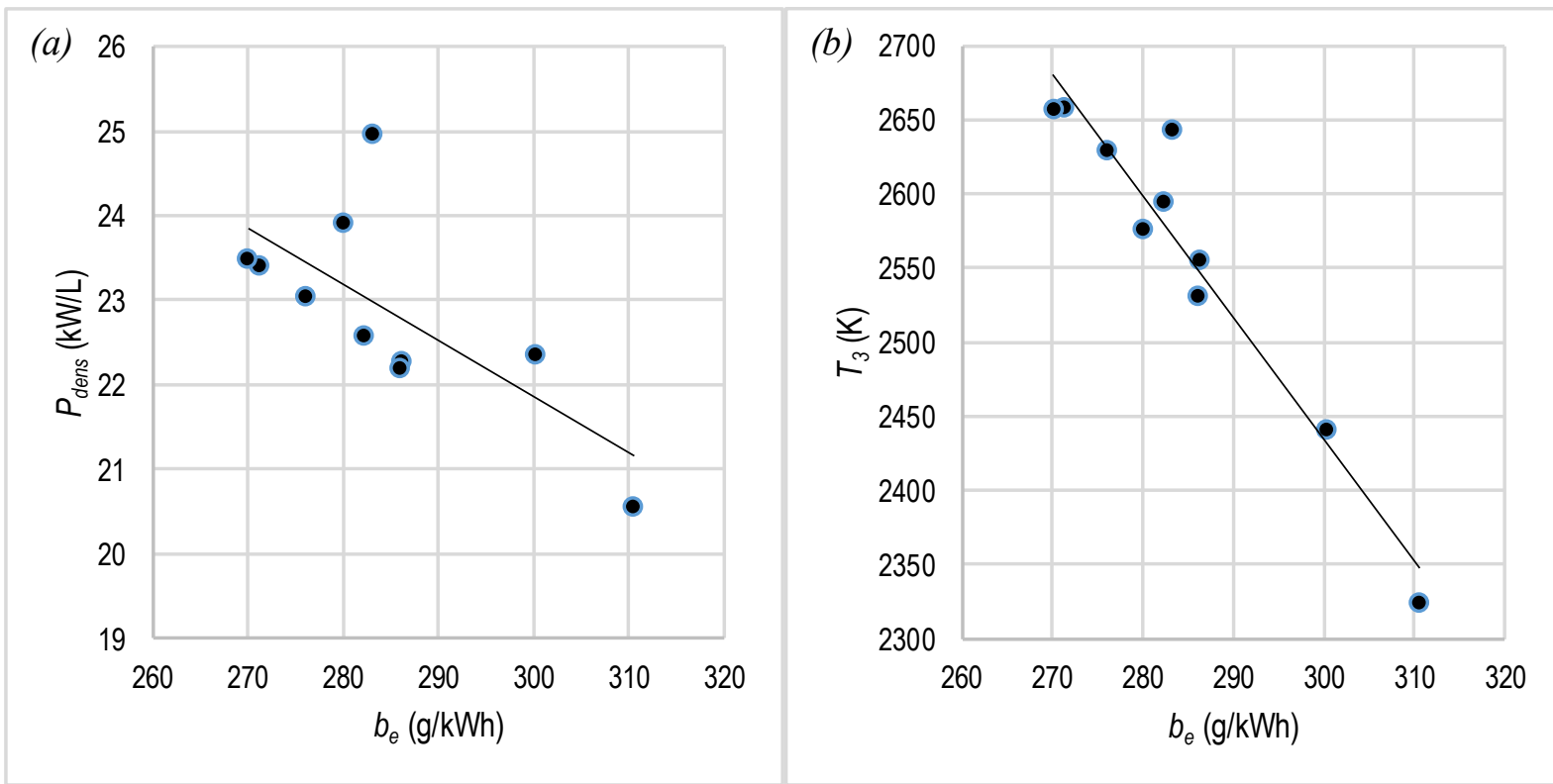

Şekil 5. (a) Gü̧ yoğunluğunun ve (b) maksimum sıcakllğın be 'ye bağglı değişimi

\section{Sonuç}

$\mathrm{Bu}$ çalışmada sıkıştırma oranı, kurs oranı, kurs hacmi ve çıkış gücü birbirinden farklı benzinli genel amaçlı motorların performanslarının karşılaştırılması için basit bir termodinamik model oluşturulmuștur. Bununla birlikte giriș sıcaklığı, 1sı transferi, sürtünme gibi parametreler için alt modeller oluşturulmuştur. $\mathrm{Bu}$ model için termodinamiğin birinci yasası ve sonlu zaman termodinamiği kabulleri kullanılmıştır. Ayrıca özgül 1sı değerinin, sıcaklığın logaritmik fonksiyonu olarak değiştiği kabul edilmiştir. Termodinamik modele göre hesaplanan motorlara ait çıkış gücü değerleri, katalog değerlerine göre karşılaştırılarak modelin kullanılabilirliği kontrol edilmiştir. Daha sonra her bir referans motor için 1sı balansı oluşturulmuştur. Isı balansında kayıp gücün, egzoz, sürtünme ve 1sı transferi ile kaybedilen güçlerin toplamı olduğu varsayılmıştır ve termodinamik model yardımıyla bu kayıp güçlerin ayrı ayrı oranları hesaplanmıştır. Motorlar arasında marka ve modelden bağımsız olarak kurs oranı, sıkıştırma oranı, kurs hacmi, gibi fiziksel özelliklerine göre karşılaştırma yapılabilmesi için kurs oranı ve özgül yakıt tüketiminin güç yoğunluğu, maksimum sıcaklık ve mekanik verime etkisi incelenmiştir. Buna göre kurs oranının artmasıyla birlikte maksimum çevrim sıcaklığı, mekanik verim ve güç yoğunluğunun azaldığı, özgül yakıt tüketiminin arttı̆̆ görülmüştür. Yapılan çalışma neticesinde özgül yakıt tüketimi ile maksimum çevrim sıcaklığı arasında, kurs oranı ile maksimum çevrim sıcaklığı arasında ve kurs oranı ile özgül yakıt tüketimi arasında güçlü bir ilişki olduğu ve oluşturulan modelin benzinli genel amaçlı motorların performanslarının karşılaş̧ırılması için kullanılabilir nitelikte olduğu görülmüştür.

\section{Açıklama}

Bu çalışma 13-15 Aralık 2018 tarihleri arasında gerçekleştirilen "International Conference on Technology and Science"da "A new approach to the determination of heat balance in general purpose gasoline engines" başlığı ile yazar Emre Arabacı tarafindan sözlü olarak sunulan bildiriden geliştirilerek ve zenginleştirilerek hazırlanmıştır. 


\section{Kaynakça}

Ansermet, J. P., \& Brechet, S. D. (2019). Principles of Thermodynamics. Cambridge University Press.

Arabaci, E. (2018a). Artık gaz kesri ve kurs oranının tersinmez Otto çevriminin performansına ve entropi üretimine etkileri. Avrupa Bilim ve Teknoloji Dergisi, (14), 83-89.

Arabaci, E. (2018b). A new approach to the determination of heat balance in general purpose gasoline engines. International Conference on Technology and Science. Retrieved January 01, 2019, from http://dergipark.gov.tr/sjmakeu/page/5766

Chase Jr, M. W. (1998). NIST-JANAF thermochemical tables. J. Phys. Chem. Ref. Data, Monograph, 9.

Diesel, R. (1898). U.S. Patent No. US608845A. Washington, DC: U.S. Patent and Trademark Office.

Dobrucali, E. (2016). The effects of the engine design and running parameters on the performance of a Otto-Miller Cycle engine. Energy, 103, 119-126.

Ebrahimi, R. (2011). Effects of mean piston speed, equivalence ratio and cylinder wall temperature on performance of an Atkinson engine. Mathematical and Computer Modelling, 53(5-6), 1289-1297.

Ebrahimi, R. (2012). Performance analysis of an irreversible Miller cycle with considerations of relative air-fuel ratio and stroke length. Applied Mathematical Modelling, 36(9), 4073-4079.

Ebrahimi, R. (2013). Thermodynamic Modeling of an Atkinson Cycle with respect to Relative Air-Fuel Ratio, Fuel Mass Flow Rate and Residual Gases. Acta Physica Polonica, A., 124(1).

Ebrahimi, R. (2016). Second law analysis on an air-standard Miller engine. exergy, 2, 17.

Ebrahimi, R., \& Dehkordi, N. S. (2018). Effects of design and operating parameters on entropy generation of a dual cycle. Journal of Thermal Analysis and Calorimetry, 133(3), 1609-1616.

Ebrahimi, R., \& Sherafati, M. (2013). Thermodynamic simulation of performance of a dual cycle with stroke length and volumetric efficiency. Journal of thermal analysis and calorimetry, 111(1), 951-957.

Ganesan, V. (2012). Internal combustion engines. New York: McGraw-Hill.

Ge, Y. L., Chen, L., \& Sun, F. R. (2008). Finite-time thermodynamic modelling and analysis of an irreversible diesel cycle. Proceedings of the Institution of Mechanical Engineers, Part D: Journal of Automobile Engineering, 222(5), 887-894.

Ge, Y., Chen, L., \& Qin, X. (2018). Effect of specific heat variations on irreversible Otto cycle performance. International Journal of Heat and Mass Transfer, 122, 403-409.

Ge, Y., Chen, L., \& Sun, F. (2009). Finite-time thermodynamic modeling and analysis for an irreversible Dual cycle. Mathematical and Computer Modelling, 50(1-2), 101-108.

Ge, Y., Chen, L., Qin, X., \& Xie, Z. (2017). Exergy-based ecological performance of an irreversible Otto cycle with temperature-linearrelation variable specific heat of working fluid. The European Physical Journal Plus, 132(5), 209.

Ge, Y., Chen, L., Sun, F., \& Wu, C. (2005). Effects of heat transfer and friction on the performance of an irreversible air-standard Miller cycle. International Communications in Heat and Mass Transfer, 32(8), 1045-1056.

Gonca, G. (2017). Performance Analysis of an Atkinson Cycle Engine under Effective Power and Effective Power Density Conditions. Acta Physica Polonica, A., 132(4).

Gonca, G., \& Sahin, B. (2016). The influences of the engine design and operating parameters on the performance of a turbocharged and steam injected diesel engine running with the Miller cycle. Applied Mathematical Modelling, 40(5-6), 3764-3782.

Gonca, G., Sahin, B., \& Ust, Y. (2013). Performance maps for an air-standard irreversible Dual-Miller cycle (DMC) with late inlet valve closing (LIVC) version. Energy, 54, 285-290.

Heywood, J. B. (2018). Internal combustion engines. New York: McGraw-Hill.

Hohenberg, G. F. (1979). Advanced approaches for heat transfer calculations (No. 790825). SAE Technical paper.

Kaushik, S. C., Tyagi, S. K., \& Kumar, P. (2017). Finite time thermodynamics of power and refrigeration cycles. Springer International Publishing.

Otto, N. A. (1887). U.S. Patent No. US365701A. Washington, DC: U.S. Patent and Trademark Office.

Özdemir, A. O., Kılıç, B., Arabacı, E., \& Orman, R. Ç. (2018). Effect of mean piston speed and residual gas fraction on performance of a four-stroke irreversible Otto cycle engine. Scientific Journal of Mehmet Akif Ersoy University, 1(1), 6-12. 
Wu, C. (2002). Intelligent computer based engineering thermodynamics and cycle analysis. New York: Nova Science.

Wu, Z., Chen, L., Ge, Y., \& Sun, F. (2017). Power, efficiency, ecological function and ecological coefficient of performance of an irreversible Dual-Miller cycle (DMC) with nonlinear variable specific heat ratio of working fluid. The European Physical Journal Plus, 132(5), 203.

Wu, Z., Chen, L., Ge, Y., \& Sun, F. (2018). Thermodynamic optimization for an air-standard irreversible Dual-Miller cycle with linearly variable specific heat ratio of working fluid. International Journal of Heat and Mass Transfer, 124, 46-57.

You, J., Chen, L., Wu, Z., \& Sun, F. (2018). Thermodynamic performance of Dual-Miller cycle (DMC) with polytropic processes based on power output, thermal efficiency and ecological function. Science China Technological Sciences, 61(3), 453-463. 